\title{
Ambiances
}

anbiances Environnement sensible, architecture et espace urbain Comptes-rendus | 2017

\section{Tragédie à Volos.}

Au sujet des ambiances, et s'il y a lieu d'y voir une pensée hospitalière de l'architecture et de la ville

\section{Clotilde Félix-Fromentin}

\section{OpenEdition}

\section{Journals}

\section{Édition électronique}

URL : http://journals.openedition.org/ambiances/850

DOI : 10.4000/ambiances.850

ISSN : 2266-839X

\section{Éditeur :}

Direction Générale des Patrimoines - DAPA - MCC, UMR 1563 - Ambiances Architectures Urbanités (AAU)

\section{Référence électronique}

Clotilde Félix-Fromentin, «Tragédie à Volos. », Ambiances [En ligne], Comptes-rendus, mis en ligne le 10 avril 2017, consulté le 23 septembre 2020. URL : http://journals.openedition.org/ambiances/850 DOI : https://doi.org/10.4000/ambiances.850

Ce document a été généré automatiquement le 23 septembre 2020.

\section{c) (i) $(9)$}

Ambiances is licensed under a Creative Commons Attribution-NonCommercial-NoDerivatives 4.0 International License. 


\section{Tragédie à Volos.}

Au sujet des ambiances, et s'il y a lieu d'y voir une pensée hospitalière de l'architecture et de la ville

\section{Clotilde Félix-Fromentin}

\section{RÉFÉRENCE}

Nicolas Rémy \& Nicolas Tixier (dir.). 2016. Ambiances, demain / Ambiances, tomorrow |

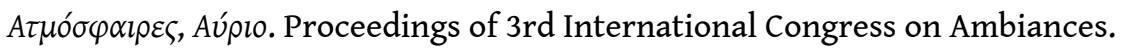
International Ambiances Network \& University of Thessaly. 2 volumes. 1016 pages.

1 J'étais à Volos pour le $3^{e}$ Congrès international sur les Ambiances, Ambiances, Tomorrow, du 21 au 24 septembre 2016. Je ne connaissais des ambiances que ce que de rares appels ou articles diffusent au-delà de ce réseau et je me permettais, avec ce bagage minime, d'être sceptique autant face à l'immatérialité de l'objet de recherche - le monde sensible -, que face à la maîtrise ou design des milieux ambiants qu'il visait. D'ailleurs, c'est la critique d'un projet de captation numériquement assisté de l'habitabilité de la ville qui m'avait amenée à proposer une communication pour le congrès. Aussi, je cultivais ces idées - et ce « je » se fait représentatif d'un point de vue extérieur -, alors que je côtoyais de temps à autre certains acteurs des ambiances, mais n'avais pas pris le temps d'en discuter avec eux.

2 Eh bien, je fus étonnée, séduite, bousculée par ce séjour. Il me plongea progressivement dans un trouble suffisant pour que je ressente le besoin d'écrire pour réfléchir, ou en tout cas pour fixer ces agitations de mon esprit ${ }^{2}$. Je ne savais quel mot mettre sur ce dérangement ; mais l'invitation de Catherine Grout ${ }^{3}$ à venir m'en entretenir à l'occasion d'une séance de séminaire du projet de recherche "L'hôpital comme milieu ${ }^{4}$ me conduisit à lui essayer le qualificatif d'hospitalier. Ma catharsis témoignerait-elle d'un statut des ambiances comme hôpital de la pensée pour la conception architecturale et urbaine? Elles me sont en tout cas apparues comme un champ alternatif, tourné vers ces territoires actuels qui ne se satisfont pas des méthodes de pensées et de pratiques courantes, comme l'hôpital en est sans doute. 
3 Afin de partager cette proposition qui fait de la notion d'hospitalité un prétexte à commentaire épistémologique, je livre ci-après mon ressenti sur cette rencontre: ressenti situé, donc local et ponctuel. Ce texte ne tente pas de rendre compte exhaustivement de ce que j'ai vécu, mais il pointe plutôt ce qui a attiré mon attention de néophyte. Ce sentiment est affecté par une maîtrise relative des langues étrangères les congressistes communiquaient dans trois langues : l'anglais, le français et le grec -, qui eut pour effet positif de me placer dans une réception singulièrement attentive aux façons de communiquer, aux attitudes, aux intonations, aux gestes, aux efforts ; ce qui n'est sans doute pas inintéressant pour notre sujet.

4 Pour des raisons liées tant au contexte qu'à la structure et à la nature des événements, mon propos est organisé selon la poétique de la tragédie grecque, alternant les scènes des personnages principaux (prologue et épisodes) et les interventions du chœur (parades et stasima), l'ensemble étant encadré par un liminaire et un exode.

\section{Liminaire}

5 Des gens arrivent de toutes parts au seuil du bâtiment de l'école d'architecture de l'université polytechnique de Thessalie. Comme eux, je me mets dans la file afin de valider mon inscription. Tout m'est étranger et anonyme, on entend le bruissement des arbres dans la brise.

6 Soudain, tout en sourire arrive Nicolas Tixier, l'un des organisateurs du congrès, qui salue, embrasse, souhaite la bienvenue. Il me présente à ma voisine et l'attente se transforme en aimable chahut. L'instant d'après, la file se démantèle et les corps glissent entre les blocs pour rejoindre l'amphithéâtre.

\section{Prologue}

7 "Ainsi, toutes choses ont leur part de souffle et d'odeurs». Jean-Paul Thibaud, fondateur du Réseau International Ambiances, cite Empédocle pour lancer la conférence d'ouverture intitulée "Le devenir ambiant $»^{5}$. La conférence entend nous donner quelques pistes, dit-il, pour s'orienter dans le champ des ambiances : depuis les multiples contextes où les ambiances sont mises en œuvre aujourd'hui, jusqu'aux paradigmes susceptibles de convenir à leurs natures. La précaution est fort utile en effet au néophyte que je suis afin de lui offrir des repères dans ce vaste panorama.

8 Je retiens au premier chef cette tentative de définition, exercice récurrent semble-t-il tant la difficulté est ontologique. L'ambiance est ici proposée comme étant "ton", « pont » et « fond », comme trois versants d'un même objet.

9 L'ambiance est «ton » (attunement), elle est «ce quelque chose » dont parlait Virginia Woolf, une coloration du quotidien qui en accentue la résonance intérieure. L'ambiance, également, est " pont» (in-between), elle est dans l'intervalle, dans le flux entre les choses, entre l'objet et le sujet, et à cet égard sa saisie est de l'ordre de l'interception plus que de la perception. L'ambiance enfin est «fond» (background) en ce qu'elle constitue la base continue disséminée de notre expérience quotidienne, faite de savoirs tacites, de gestes fantômes et de chorégraphies collectives. Une courte vidéo est projetée pour éclairer le propos: des gens sortant d'un cinéma s'arrêtent pour échanger sur le trottoir à la lueur bleuie de la façade et des voitures qui passent. Elle 
illustre le caractère non-représentationnel de l'ambiance quand il s'agit plutôt d'effets de présence.

De fait, l'ambiance ne relève pas de l'essentialisme, commente notre orateur. Elle n'est pas une essence détachée de l'existence. Elle procède davantage d'une "effectuation » que d'une détermination, c'est-à-dire qu'elle met l'accent sur les moyens et leurs effets plutôt que sur les fins; elle n'en recèle pas moins de puissances, au contraire. Ses modes opératoires sont diffus et distribués, impersonnels et imperceptibles, via des microphénomènes enchâssés les uns dans les autres. Ils procèdent par inclusion et infiltration. Sa consistance est de l'ordre de l'infra, précise-t-il. Elle est infrasémiotique, en-deçà des signes, infra-verbale, en-deçà des mots, infra-cognitive, endeçà des savoirs. Mais cette basse intensité n'en a pas moins d'effets éthiques et politiques puissants quant à sa manière de nous toucher. De la sorte, l'ambiance concerne le concept même de l'habiter : elle a le pouvoir de susciter le «se sentir chez soi $»$... ou bien de discipliner les corps et de normaliser les conduites.

11 Toutefois, pour se tenir dans une politique esthétique des ambiances, sa conception est délicate voire revêche car une ambiance ne se décrète pas, et n'est jamais chose faite une fois pour toute. Il est possible seulement d'accompagner son émergence, c'est-àdire de mettre en place les conditions idoines à seconder le monde en train de se faire, comme l'exprimait Franz Kafka.

12 En réalité, conclut Jean-Paul Thibaud, l'ambiance trouve des axes d'intelligibilité dans plusieurs paradigmes soutenant les mutations du monde actuel. Et le voilà qui cite sans nous ménager : le tournant de la nouvelle phénoménologie, la visée pragmatique avec le moyen de l'enquête, la théorie non-représentationnelle poststructuraliste portée sur les affects pré-individuels, et enfin la théorie sphérologique, climatique et immunologique de l'habitabilité.

13 Certes, ces tendances ne sont pas disjointes, mais tout de même, me suis-je demandée, que penser d'un tel cosmopolitisme? D'une telle absence, assumée, de positionnement? Finalement, j'entends que c'est plus globalement dans le tournant de la sensibilité que l'ambiance se loge : quand le sensible remonte à la surface, il trouve en l'ambiance son plus puissant porte-voix, dit Jean-Paul Thibaud en substance. Et elle y œuvre en mode infra-sensible, en-deçà des sensations, esquissant un nouveau cadre pour un régime d'affectivité qu'il veut garder pluriel.

14 Tout ceci me donna alors le sentiment d'un déplacement. Nous voilà hors des champs disciplinaires, et je réalise que jamais, ou si peu, les mots architecture, urbanisme, paysage, design ne furent prononcés. Nous voilà consécutivement hors des concepts qui leur sont attachés, et même hors des grandes tournures qui servent d'habitude à la réflexion pour se formaliser. Ici, des liens furent faits avec la littérature, qui n'eurent pas besoin de s'énoncer et de se justifier. Cela se ressent finalement comme un nettoyage, une économie de la pensée.

15 Mais surtout, cette conférence eut pour effet d'ébranler mes a priori quant aux ambiances puisque les perspectives - qui me laissaient sceptique - d'une esthétisation du cadre urbain adossée à un marketing sensoriel, dans le cadre d'une économie capitaliste de l'expérience, et soutenue par le tournant machinique de la perception, l'assistance technologique de l'attention et la dissémination des moyens numériques... furent constatées mais non agréées. Ce design d'ambiances peut en effet affecter profondément la relation à la ville en générant - et Jean-Paul Thibaud convoque à présent Gilbert Simondon - une dissociation des «milieux associés ", un découplage 
entre les producteurs et les consommateurs, disons entre les designers et les usagers. C'est pour moi un renversement de ce qui me semblait animer les ambiances, c'est une péripétie au sens grec, une phase imprévue du drame qui fait passer d'un état à son contraire.

Mais l'ambition de cette annonce en prologue n'était-elle pas à entendre ainsi: quelques pistes qui (ré-)orientent le devenir? Est-ce un virage? En tout cas, la voix protagoniste de Jean-Paul Thibaud sera citée régulièrement par les participants lors du congrès, comme une voie à suivre.

Le décor est planté, et il m'est hospitalier comme : engageant.

\section{Première parade}

Plusieurs sessions débutent à présent en divers lieux de l'université, et je me dirige vers celle qui est intitulée « Théorie, Critique, Politique ».

Sensibilisée sans doute par ma péripétie, j'apprécie d'un premier intervenant ${ }^{6}$ l'expression d'une position réservée à l'égard des technologies ambiantales du pouvoir appliquées à l'aménagement des milieux de vie, urbains et quotidiens (c'est une place de ville qui sert d'exemple) et la tendance récente à les lisser. La théorie biopolitique de Michel Foucault, resituée par rapport à celle du Umwelt de Jakob von Uexküll, permet de révéler l'autorité paradoxalement contenue dans cette "aisance, propreté et monotonie ", pour parodier cette fois le triptyque de Charles Baudelaire.

Je remarque le soulèvement du public de notre petite salle quand une personne fait remarquer qu'il vaut pourtant mieux, si l'on prend cet exemple, des cafés silencieux que des cafés avec une musique donnée qui représente un régime technologique imposé. Non! On rétorque à cela que le clivage entre la technologie et le régime du vivant n'est plus possible aujourd'hui, qu'il est un raccourci dualiste. Les outils « sociotechniques » et « techno-sensoriels » sont présents au quotidien, il ne sert à rien de les nier ou de s'y opposer. Une telle attitude est de celles qui empêchent de « construire le commun", d'élaborer à partir du réel et de nos subjectivités singulières, une communauté, un « vivre en commun » des différences. Comme je l'entendrai plus loin, il existe des "artificialités positives» et des «technologies bienveillantes", et il vaut mieux regarder dans cette direction. Encore plus tard, au sujet de la participation des ambiances comme moyen d'action publique, il sera question de s'opposer aux formes radicales de soulèvement qui n'engendrent qu'un commentaire stérile et un durcissement des ripostes.

21 J'en déduis une position accommodante des ambiances, qui m'interroge par rapport au retour plus général de la critique. Comment faire que la conciliance ne soit pas complaisance? C'est pourquoi j'attrape cette précaution au vol d'une discussion enflammée : si la question des ambiances est le terrain idéal pour entretenir l'incertain et l'informel, elle risque l'anarchie sur le terrain socio-politique. Le mode d'être de cette forme de puissance appelle d'autant plus à ce que l'assemblée se dote d'une constitution au sens du politique, c'est-à-dire que son organisation soit réglée, ses actions discutées, ses espaces distribués. La part laissée à l'indétermination n'empêche donc pas une manière d'ordonnancement et de construction. 


\section{Seconde parade}

22 Me voici désormais dans la session "Héritage du futur" et cet oxymoron est symptomatique, il me semble, de la position disciplinaire des ambiances: de l'histoire, certes, mais discutée avec le présent et orientée vers un à-venir afin de viser comment les ambiances peuvent devenir les instruments de compréhension des actions de patrimonialisation.

23 La tonalité y est plus douce et studieuse. On y échange et on disserte sur les méthodes historiographiques. Celles-ci sont très ouvertes, du dépouillement de brochures commerciales à la collection de portraits de la vie quotidienne, à la lecture de romans, au déchiffrage d'une ville comprise comme un palimpseste.

24 Je reste dubitative (et ne suis pas la seule) devant une étude de textes littéraires à partir de l'analyse statistique de mots-clés, dont les contenus latents amènent des interprétations personnelles contestables. Et je reste sur ma faim quand les observations rejoignent la problématique du "style». Mais je suis emportée l'instant d'après par l'expression motivée et motivante d'une démarche de projet $^{7}$ de régénération urbaine à partir de ses layers (couches d'ambiances enracinées ou émergentes). La ville est approchée, en se référant historiquement à Patrick Geddes, comme la stratification d'une mémoire naturelle (réseau hydraulique, données topographiques et biologiques, localisation des friches...) et d'une mémoire urbaine (patrimoine matériel et immatériel, évolution des usages, relevé des empreintes de l'histoire sociale, parole des habitants), qu'il s'agit de recomposer, puis de réactiver, en établissant avec les habitants des "cartographies de désirs" et des protocoles d'« usages extensibles ».

25 Il y a quelque chose de très plaisant dans ce travail - qui, de mon point de vue néophyte, aurait pu ignorer la notion d'ambiance - en ce qu'il illustre, d'une part comment la pluralité de l'idée d'ambiance parvient à épaissir le réel (par des détails, des parts d'invisible, des histoires de vie, d'autres formes de vie), d'autre part comment elle maintient, en contraste avec la somme des connaissances accumulée, une volonté de projet qui se tient aux micro-interventions habitantes (ou conservative surgery selon l'analogie geddesienne à la thérapeutique).

Le café est un art grec - qui le sait? - qui conserve ici toute sa valeur sociale et se décline, dans ses boissons et ses agréments, tout au long de la journée. De nombreuses pauses ponctuent agréablement le congrès, où je constate, loin des mondanités habituelles, une propension facile à la discussion malgré le choc des langues et des provenances. Il m'apparaît qu'elle est aidée par la pluridisciplinarité qui prévaut décidément. Elle suscite les échanges et elle les rend avides, sans crainte d'aborder des territoires loin du sien ou d'être dérangé par de l'inédit : l'ambiance nous rejoint. C'est pour moi la première fois que j'observe une dynamique véritablement libérée et transversale par rapport aux disciplines; et consécutivement je constate ses effets stimulants et ses bénéfices pour la recherche. Une culture commune est rendue possible et espérée.

27 Toutefois, n'est-ce pas au risque d'un manque d'approfondissement? D'un éclectisme? À couvrir des champs divers et distincts, ne risque-t-on pas de manquer de la précision experte de l'approche scientifique? Quelque chose, en effet, m'a jusqu'ici semblé se 
tenir au seuil du raisonnement. Mais après tout, les ambiances se tiennent au seuil du sensible...

Est-ce là le sort, ou plutôt une condition, de la pensée des ambiances ? Se tenir hors de la spécialisation pour préférer l'épaississement qu'une compréhension pluridisciplinaire, polylingue, autorise? Après tout, l'éclectisme est un art philosophique grec qui sut combiner toutes les catégories, sans exclusivité ni hiérarchie.

\section{Premier épisode}

François Laplantine entre à présent en scène pour évoquer, à son tour, ce que lui a inspiré le thème "Ambiances, Demain»: l'idée de transformations esthétiques consécutivement à une aporie du langage.

Notre deutéragoniste n'est pas du réseau, il est un anthropologue des modalités du sensible, où le verbe tient une place cruciale puisque nos catégories de pensées sont aussi des catégories de langage. Or, il commence en pointant ces cinq mots qui, dans les langues européennes, représentent des réalités voisines: milieu, environnement, ambiance, atmosphère, paysage. Le voilà dès lors, en écho aux propos de Jean-Paul Thibaud, amener à son tour cette proposition de définition : l'ambiance est milieu $\mathrm{x}$ moment $\mathrm{x}$ sensation, c'est-à-dire un milieu (compris comme espace micro-local), à un instant $\mathrm{T}$ (au temps présent lié à une présence), produisant telles ou telles sensations (écoute corporelle ressenti par un « je » qui est un «nous »).

31 En effet, l'ambiance est nécessairement "chorale », vécue ensemble, et affecte un milieu en élaborant comme un lien dans un lieu. Elle est une réalité indivise qui n'est pas de l'ordre de l'objet, ni même d'une somme d'effets d'objets (lumières + sons + formes, etc.). Elle échappe pour cela à l'observation et demeure rebelle à la verbalisation. Elle est objectale mais en englobant le sujet et en suspendant ainsi la séparation sujet-objet. Elle appelle consécutivement un langage non égologique, tel que le « il y a » d'Emmanuel Levinas par exemple. Il advient... Il survient... Il arrive... Il y a de l'amour dans l'air, dira-t-on, au lieu de je t'aime...

Bref, l'orateur expose toutes les difficultés et les paradoxes que l'ambiance pose à notre pensée occidentale, et il s'en va chercher le point de vue grec (où l'aesthesis renvoie à la capacité de se sentir concerné) et l'entendement japonais (où le contexte intègre le sujet) car plus à même, selon lui, de cerner le phénomène. L'expérience de l'ambiance nécessite en effet une transformation problématique de la subjectivité, qui se replie sur la relation entre être et lieu. Ce faisant, elle absout la fonctionnalité : en effet, sauf ses versions consuméristes grégaires qu'il met lui aussi de côté, une ambiance ne sert à rien. Elle abolit la préméditation: une ambiance n'est ni focalisée, ni finalisée; elle outrepasse l'appropriation et même la direction (dans le sens vectoriel). Elle invite en revanche à s'intéresser à nouveaux frais à la notion de limite: dans le temps (temporalité), dans l'espace (spatialité et idée de seuil), relativement à l'attention (disponibilité), etc.

33 Mais ce phénomène, qui met profondément nos schèmes en examen, ne concerne pas seulement la question de la conception des ambiances. Il est, précise François Laplantine, une réaction idoine et nécessaire face au présent, alors que la globalisation génère une uniformisation des identités, qui tend à engendrer son contraire, c'est-à- 
dire une fragmentation différentialiste voire intégriste, un durcissement identitaire. L'orateur ne peut que constater les limites actuelles du langage et des sciences humaines à rendre compte et à trouver du sens à la situation. Il en résulte de la méfiance entre nous, voire une dépression généralisée (financière et morale) et la place vacante laissée aux ambiances anonymes normalisées. La pensée grecque et sa logique triadique, ou la pensée japonaise et sa plasticité inouïe, en ce qu'elles permettent une oscillation et une pluralité contingente plutôt que ces extrémités manichéennes, restent dès lors des territoires à découvrir.

Alors que je relis mes notes pour écrire ces lignes, je revis intérieurement l'état dans lequel cette conférence me mit : l'impression, réjouissante, que les ambiances recèlent un potentiel de renaissance.

Il ne s'agit pas de résoudre les problèmes du monde. Il ne s'agit pas de faire table rase et d'innover (de projeter du nouveau). Mais il s'agit de détecter et nourrir à chaque instant des modes indiciels et changeants de relation au monde. Et puis ceci : le «je» qui est un «nous » y peut quelque chose, n'est-ce pas régénérant ?

Il est temps pour François Laplantine de conclure, en l'occurrence de nous mettre au travail. À partir d'une relecture de cette formule de Wittgenstein: «Ce que l'on ne saurait dire, il convient de le taire", il propose «Ce que l'on ne saurait dire, nous pouvons le montrer ». Et d'inviter à transformer performativement la situation, au sens de la linguistique de John Langshaw Austin, au sens des arts du théâtre ou des arts vivants, par des gestes, des sons, des matériaux, des images : ce que permet, rappelle-til, l'architecture! Avant de faire cette suggestion complémentaire: "Ce qu'on peut montrer, il faut tenter de le dire ». Contre la démission intellectuelle qui laisse la place libre aux conformismes, il est nécessaire d'articuler l'expérience, de la dire, de la théoriser.

Il en est comme si notre deutéragoniste répondait au protagoniste : «Grande, belle ton annonce, Thibaud, mais quel logos, quelle logique pour conduire et aménager l'infrasensible?»

Si cela me fut hospitalier? Oui, comme impliquant.

\section{Stasimon} Peter Sloterdijk, du concept méso-poétique et atmo-topique d'«île», considérée comme une forme de découpage du sensible. L'insularité est, selon le philosophe, une condition d'être-au-monde qui pousse l'homme à aménager son habitat, c'est-à-dire à créer entre les êtres et leur environnement une sphère intermédiaire de solutions ou d'artefacts de mise à distance, lui permettant de quitter la prison de l'adaptation corporelle et de faire naître le corps sensible et intime. Cette approche des limites, atypique et controversée, a un potentiel pour nourrir les ambiances en ce qu'elle mêle physicalité et sensualité, mais aussi en ce qu'elle dévoile le paradoxe entre isolement et connexion. Une ambiance isole-t-elle ou relie-t-elle?

La session semble avoir été organisée autour de cette problématique, et je comprends que c'est un point sourcilleux pour les ambiances. La salle s'embrase d'ailleurs contre cette idée d'une intériorité de l'habiter entendue comme encapsulation, et je relève la 
difficulté qu'a le public à entendre la réponse de l'intéressée. Dans ce besoin de limite, explique-t-elle, il s'agit d'inclusion plutôt que d'isolement, qui n'est jamais exclusion.

Soudain, je réalise dans quelle quête d'objectivité la question des ambiances et la constitution de son réseau ont cherché à asseoir leur crédibilité, ici malmenée. Reconnaître aux ambiances une part subjective fait craindre ce que la subjectivité enferme d'arbitraire mais aussi de culpabilité morale, de psychologisme futile. Une position intermédiaire entre l'objectif et le subjectif est sans nul doute à trouver, qui explique ces agitations.

Plus tard, deux intervenantes ${ }^{9}$ venant de Rio de Janeiro, chercheures dans un laboratoire brésilien qui a pris la mesure de ce bouleversement et se nomme même courageusement "Architecture, Subjectivité, Culture », posent les bases d'un concept d'«empathie spatiale ». Ce concept m'apparaît très pertinent pour évoquer la réciprocité d'une relation entre l'espace (tiens, c'est la première fois que j'entends ce mot) et le corps, relation qui n'est pas d'appartenance, mais d'expérience. Par la somme des références listées, de l'Einfühlen de Theodor Vischer au corps ego- et allo-centré d'Alain Berthoz, en passant par l'espace phénoménologique de Merleau-Ponty et la cognition incarnée de Francisco Varela - et j'en passe - on sent le besoin d'assurer sa position avant d'en arriver à l'idée d'un « corps élargi » par le champ perceptif.

Je les interroge sur la possibilité d'envisager une nouvelle subjectivité, non plus moderne et clivée, mais ambiantale justement : un «sujet ambiant» dont les limites propres sont redéfinies par le rapport au milieu. En effet, nous nous entendons làdessus, en même temps que je constate que cette entrée du subjectif, quoique aménagée et prévenante, laisse de nombreux sceptiques dans la salle.

Pourtant, une autre session ${ }^{10}$ se penche à ce même moment, et de façon multiple et aventureuse, sur la mise en expérience et l'expérimentation matérielle des ambiances. Celles-ci reposent sur une implication individuelle de plus en plus complète, phénoménologique souvent, d'autant plus que l'on s'oriente du sensible vers l'infrasensible. Depuis celle du transect, des approches inédites sont testées: l'atelier d'écriture, l'immersion corporelle suivie de maquette mentale, l'art de la fête, la transcription scénographique incarnée, l'immersion, la diffusion d'odeurs, la notation des rythmes, etc. Toutefois, il est à relever qu'elles restent majoritairement dans une visée descriptive ou projective, davantage que diagnostique ou propositionnelle. Du renouveau en tout cas, qui me donne désormais à voir ces chercheurs comme des « ambiants » (qui rime drôlement, permettez-moi, avec « déviant », « divergent » ... ou «impénitent »).

Je relève la simplicité avec laquelle de telles questions furent posées. Je réalise d'ailleurs que depuis le début du séjour, je ne me suis jamais sentie étrangère aux préoccupations, aussi diverses étaient-elles. Sans doute, cela vient-il du fait qu'elles se rapportaient chaque fois à quelque chose que l'on partage tous, intimement, quotidiennement : le réel, celui que l'on a effectivement vécu, ou que l'on aurait pu vivre.

48 L'instant d'après, je prends un café en discutant avec un jeune homme prénommé Appolos, alors en MBA à l'université, qui suit certains moments du congrès par curiosité. «Je suis du pays où l'on pleure, me dit-il, parce qu'on n'a pas d'argent ». Faisant miennes les quelques 48 heures passées sur place, je commençais justement à prendre la mesure du cadre, du climat, des gens, du fonctionnement local. L'atmosphère ici, à Volos, n'a rien de pleurnicharde. Au contraire, je lui trouve quelque 
chose de riche dans sa façon d'être hospitalière modestement. Beaucoup de bâtiments abandonnés par leurs habitants, de nombreuses constructions arrêtées à l'état de chantier, et la subsistance relative des infrastructures, donnent à penser que quelque chose s'est arrêté... mais j'ajoute: à temps. Car cette suspension a quelque chose d'heureux, de l'ordre d'une libération, comme survenue avant un débordement, un excès de maîtrise ; celui, peut-être, qui m'accable en France. Hormis la zone touristique, la ville que j'ai eu l'occasion de sillonner à vélo présente maintes structures en béton (de type Domino), laissées telles quelles. Elles donnent envie de s'y glisser et de les habiter quelque temps ainsi; après tout, il ne manque que d'y aménager quelques limites matérielles. Le paysage urbain qui en résulte est poreux, aéré, et marqué par le temps et les événements. Sa vacance m'est accueillante, comme, apparemment, à nombre d'étudiants et de chiens errants.

Le site de l'université semble un grand terrain vague dont on ne perçoit les clôtures que de l'extérieur, où des édifices sans intérêt (qui n'attirent pas l'intérêt) sont simplement posés et laissent une large place à des allées, une végétation sauvage, des murets et des recoins, des groupes de chaises et un théâtre extérieur. C'est un lieu fortuné en ce sens qu'il offre plein de milieux très variés. Des dialogos s'y tiennent ici ou là, son agora nous réunit certains soirs. Il y a des gens un peu partout, et l'on peut même s'isoler.

Appolos acquiesce mais précise: "on pleure parce qu'on ne sait pas où va notre argent ». Ce qui est là ne suffit pas, il faut s'intéresser à ce qui va, et vers où cela va. Les ambiances qui se définissent au présent, qui sont du pur présent : comment pensentelles le «devenir»? Plutôt que projeter comme imposer, je comprends: comment projettent-elles vers l'à-venir?

\section{Second épisode}

51 Voici le tritagoniste Marcos Novak, venu de Californie pour une conférence intitulée " Oh Ambient Demons». Il va s'avérer pour moi qu'on en tient un, un démon des ambiances, un «ambiant » authentique. Je ne connaissais de lui que le théoricien de la conception "non-standard » au tournant du XX ${ }^{e}$ et du XXI siècles, qui théorisait sur le «cyber-espace » et la "trans-architecture », et prédisait l'« éversion », c'est-à-dire l'échappée du monde virtuel dans le monde quotidien.

Après s'être lui-même présenté à travers ses derniers travaux - des versions créatives d'interaction homme-machine - il se lance fougueusement dans un récit des formations, transmutations, circonvolutions mentales dans lesquelles l'idée d'" Ambiances, Tomorrow» l'a, à son tour, emporté ; et nous avec lui désormais.

Il démarre par une curiosité étymologique autour du mot ambiance, qui suscite un glissement vers le mot démon. Puis il met les deux termes en vis-à-vis, et continue à les décliner selon leurs racines, leurs synonymies, leurs sonorités, la pluralité de leurs significations. Ce jeu de collage tend au cadavre exquis quand, pour chaque association, il livre ce que cela lui évoque, ou bien le mène à aller chercher plus loin. A l'instar d'une fouille curieuse et enfantine dans un dictionnaire qui amène l'esprit à inventer des chimères, son propos glisse doucement vers la mythologie.

Dès lors, le jeu de langage apparaît avoir éveillé un jeu de piste, puisque le voilà qui nous narre à présent la traversée, conduite et colorée par ces préliminaires, qu'il fit quelques jours avant le colloque - depuis Athènes jusqu'à Volos, en passant par Delphes 
- en quête des démons, passés et présents de la Grèce, qui font son ambiance. Il s'appuie cette fois sur ses propres photos prises lors du parcours : sa voiture de location devant une devanture brisée, la vue de sa chambre d'hôtel sur un ciel orageux, les ordures cachées dans les sites préhistoriques, la trace d'un ornement sur une colonne, la végétation qui s'y installe, un appel à manifestation épinglé sur un arbre, etc. Chacune est prétexte à disserter de figures de vie, de combats terribles, de croyances perdues. Pour finir, sans transition plus logique, il s'attarde sur la description d'une installation ambiante intitulée "Energy Parachute / Entelechy Drone» qu'il a réalisée en 2012 au Daejon Museum of Art, en Corée du Sud. Des drones téléguidés sillonnent en filmant une grande toile blanche agitée par une soufflerie. La vidéo est projetée sur les autres murs de la salle... Je réalise combien je suis incapable de transcrire cette scène avec des mots.

Face à l'étrangeté de la méthode, je confesse m'être peu à peu détachée du contenu pour apprécier le jeu de ce brainstorming, ici authentique « orage de cerveau ». Il y avait quelque chose de fascinant, d'un point de vue épistémologique, dans l'organisation de cette pensée impétueuse, qui pourrait passer, franchement, pour du « n'importe quoi ». Et en même temps, force est de constater qu'une position quant aux ambiances, et un message éthique voire politique relatif à la conception architecturale et urbaine, sont passés, et ceci grâce à l'ambiance elle-même que la conférence instaura.

Il en est comme si notre tritagoniste rétorquait au protagoniste et deutéragoniste: "C'est le mythe qui est mon logos et ma logique. Ainsi que les anciens hommes mettaient leurs actions et leur philosophie à peupler leur milieu aussi ardemment que nous-même aujourd'hui mettons les nôtres à les vider de sens ». Mythe : ce qui naît de cet esprit du "je » qui est un "nous », des origines qu'il réclame, de la suite et les dénouements dont il a soif.

Que le mythe succède à une conceptualisation de l'influence des technologies numériques sur le design de nos milieux de vie m'apparut symptomatique pour les ambiances.

\section{Stasimon}

58 Je ne pus malheureusement pas assister à la séance des questions car une session importante débutait ailleurs.

59 Une présentation ${ }^{11}$ nous emporta d'abord en promenade dans le jardin japonais Murinan de Kyoto, en vue de comprendre la structuration dynamique prégnante de son ambiance, et pour cela l'importance de faire des choix. Cette précision retint mon attention, car elle suggère un observateur actif et volontaire, et je réalise que c'est un point de vue que je n'ai pas encore entendu (à part, sur un autre mode, avec Markos Novak). Malheureusement je ne pus en apprendre davantage, la question est encore au travail pour son auteure.

60 C'est alors Jean-François Augoyard qui prit la parole au sujet de ce fait si banal et récurrent : quand une ambiance apparaît à la conscience. Quelqu'un coupa à cet instant la climatisation de notre salle (action prévue) : oh volupté soudaine, sentiment de paix que l'on voudrait durer toujours! Voilà, dit-il après quelques secondes, cela. Mais ce peut être une voix qui soudain prévaut dans un marché, un ivrogne qui se met à chanter. J'avais entendu ailleurs, dans une autre session, comment des survenues telles 
qu'une découverte fortuite, la peur, ou l'impression de se perdre, avaient été le moyen de la saisie authentique de l'ambiance d'un lieu. La notion grecque de métabole fut dès lors proposée pour en rendre compte : quand, dans un ensemble de facteurs donnés, tout change, s'échange sans cesse et se transforme dans un flottement permanent.

61 J'ai noté précieusement la tentative, forcément trop brève, de décrire ce phénomène d'apparition selon trois degrés d'intensité. Il y a d'abord le «quelque chose s'est passé »; ensuite l'épiphanie, la surprise ; et enfin l'émerveillement, entre interrogation et imagination. Quelque part entre l'ambiance objective - catégorisable - et l'ambiance inaperçue - latente -, l'« ambiance apparue » devient finalement révélatrice de la part de subjectivité des ambiances. Elle apparaît pour certains, disparaît peut-être pour d'autres, elle en laisse encore d'autres indifférents.

Des questions ont suivi afin de savoir si cette singularité pouvait être entendue dans le cadre de la conception de l'espace, c'est à dire lorsque l'on travaille sur un projet et que l'on scrute le moment où une "ambiance » cognitive, une image mentale ambiante, surgit. Face au phénomène si minuscule et enchanteur qui était décrit, la perspective de sa projettation ne donnait-elle pas à craindre une mainmise et une artificialisation de cet apparaître ? Je le crains... mais l'interrogation ne fut pas comprise, ou ne voulut pas être comprise.

63 Je dois à présent parler de mon cas puisque j'intervenais dans cette ultime session, et ce sera une seconde péripétie. Mais je vais proposer de considérer, si vous voulez bien me suivre, que le « je » dont il est question ici est plus ou moins représentatif d'un « nous », formé au projet moderne ${ }^{12}$. J'ai proposé de considérer le vêtement comme donneur et capteur naturel d'ambiances urbaines, afin d'en critiquer les versions " augmentées » par l'insertion de technologies qui suppléent au corps, et d'introduire la notion d'«ambiance portée ", que l'on porte et qui nous porte. Adossée à l'expérience mobilisatrice, poétique et politique, elle me semblait s'inscrire au cœur des débats de ces derniers jours.

$\mathrm{Au}$ travers des commentaires qui suivirent, pourtant généreux et désireux d'approcher mon territoire pour eux inédit mais pertinent, la réception fut déchirante pour moi. Car elle me fit comprendre que même si les idées nous rapprochent, ce que je manipule, ce que je communique n'est pas « ambiant ». Alors que j'adhérais de plus en plus à cette pensée alternative, à de nombreux égards séduisante, je réalise à quel point j'étais sans le savoir matérialiste, disons attachée à la matérialité; fonctionnaliste, disons non détournée des fonctions; méthodique, disons concernée par les questions de méthode et de cadre scientifique. Ce que j'avais retenu comme accueillant dans cette approche, révèle ce que je ne suis pas, mais peut-être aspire à être.

65 À ce moment-là, je vous assure que je vérifiai la pertinence de l'effet étudié un instant avant: l'ambiance de la salle disparut! Je me sens subitement plus étrangère qu'au premier jour.

Un moment après, j'échange paisiblement avec Jean-François Augoyard qui modère mon impression, et peu à peu quelque chose se restaure. Mais ce choc m'avait fait apparaître la position finalement dissidente, en effet déviante ou divergente, des ambiances - en tout cas, celle des fondateurs de cette pensée alors nombreux dans la salle - par rapport à l'architecture ou à l'espace aménagé, et aux paradigmes jouant un rôle majeur dans la conception. S'il y a dissidence, elle est sans mépris ni critique de ce 
dont elle s'écarte, elle se déploie de façon autonome. « Votre système ne nous intéresse pas, faites sans nous, nous travaillons au nôtre ", semble-t-elle dire joyeusement.

J'avais cru à une absence de méthode, ou une absence de visibilité des méthodes, tant de conception que d'intellection, avant de réaliser que celle-là même à laquelle je m'essaie, la " pratique phénoménologique ", ne s'édicte pas plus. Elle s'élabore à partir de l'expérience elle-même, comme une construction eidétique induite par la relation au milieu. Plutôt que de faire du projet d'ambiance, ne s'agit-il pas davantage d'être disponible d'un point de vue tout à la fois sensible, corporel et intellectuel, à ce qu'un milieu dégage ? Puis, de cela, avec cela, faire projet ? C'est que l'ambiance n'est pas une méthodologie de recherche en soi (mais des méthodes lui sont appliquées), non plus qu'un objet d'étude particulier (mais certains objets permettent de l'approcher). C'est un regard, une attitude autre sur les mêmes objets du monde : celle du bienveillant, de l'inquiet, de l'émerveillé... de l'ambiant.

«Tout, potentiellement, peut être étudié par ce biais », me dira plus tard ce précurseur des ambiances alors que je le croisais fugacement dans le hall de notre hôtel. Et il fit un geste de ses deux mains vers le bas, comme faisant mine de les glisser sous quelque chose. Cela m'a bien troublée une fois encore, puisqu'intuitivement j'aurais fait un geste des mains formant vers le haut une calotte.

\section{Exode}

69 Alors que le congrès s'achevait à la fin du troisième jour, il m'apparaissait comme une aurore ou un soir du temps (un seuil), pareils à ceux d'une belle journée, qui sont tout agités et illuminés de prestige par un soleil très bas sur l'horizon, et se colorent et se remplissent de formes vagues, de vapeurs et rayonnements décomposés, de grottes errantes et de monstres ou déités brillants. Quels sons doux et puissants et quelles harmonies étranges, quelles croyances, quelles images sans objets, quels gestes gracieux il m'a semblé entendre et vivre, tels qu'ils s'en dessinent à présent dans la substance de la nuit qui m'entoure?

Ils furent d'abord engageants, en ce qu'ils annonçaient, comme une porte qui s'ouvre, une écologie de la sensibilité, avec des termes et des gestes amicaux. Ils furent ensuite réjouissants, car déployant l'espoir d'une renaissance; mais également impliquants quand il faut pour cela remettre en jeu ses logiques et ses visions. Ils furent alors dérangeants, quand le jeu emporta à l'incertitude, vis-à-vis de soi-même et de ce qui advient. Alors que l'on commence à y trouver un lieu de pensée accueillant, il s'avère rétif à notre saisie. Il rappelle son altérité, renvoie à soi, ravive la nécessité de se situer par rapport à son histoire et au monde, il sollicite une position politique à l'échelle individuelle tournée vers le commun. Il oblige à nous déprendre de nous-même et à nous dépayser. En cela, il alimente une tension avec le milieu qui est une force d'existence.

71 Quelle est donc la mystérieuse raison de cet état? Ce ciel, me répondit Empédocle, ces conditions, ce qui meut les choses ici, cette atmosphère qui s'est déployée. Tu perçois son hospitalité : engageante, impliquante et positivement dérangeante. Car hospitis, selon les merveilles de l'étymologie, est celui qui donne ou reçoit de hospes, l'étranger qui est aussi l'ennemi. L'hospice est aussi accueillante que... hostile, l'hôtesse est aussi l'otage... Cette ambiguïté et cette mythologie méritaient bien une tragédie. 
Si tel est l'enjeu des ambiances comme je l'ai fantasmé, je soutiendrai volontiers le retour du mythe en architecture, soit le retour de l'hospitalité pour dire l'habitabilité.

\section{NOTES}

1. Voir la présentation du congrès sur le site du Réseau International Ambiances: http:// ambiances.net/ambiances2016. Les actes du congrès ont été publiés en deux volumes, sous la direction de Nicolas Rémy et Nicolas Tixier. Ils sont également disponibles en ligne à l'adresse : https://halshs.archives-ouvertes.fr/AMBIANCES2016/

2. Je remercie Valérie Mace, avec qui je m'en étais entretenue, de m'avoir suggérée de transmettre ces impressions.

3. Professeur d'esthétique à l'École nationale supérieure d'architecture et de paysage de Lille.

4. HoCoMi (Hôpital comme milieu) est un projet de recherche fondamentale et recherche-action sur l'architecture, le paysage, les pratiques au sein de l'hôpital comme lieu de vie dans la ville. Il est dirigé par Catherine Grout (LaCTH -Ensapl) et Céline Barrère (LAVUE-Ensapvs), et soutenu depuis 2012 par le CHRU de Lille et l'EEHU (Espace Ethique Hospitalier Universitaire). Cet article prolonge une communication faite durant le séminaire d'automne 2016 dédié à la notion d'ambiance : Clotilde Felix-Fromentin, «Au sujet des ambiances, et s'il y a milieu d'y voir une pensée hospitalière de l'architecture et de la ville », Ensapl, 16 novembre 2016.

5. Les enregistrements vidéo des conférences du congrès sont disponibles à l'adresse http:// ambiances.net/ambiances 2016

6. Il s'agit de Céline Bonicco-Donato dont l'intervention s'intitule «De l'aménagement des milieux à la technologie ambiantale du pouvoir - Eléments pour une philosophie politique des ambiances ». Consultable sur : https://hal.archives-ouvertes.fr/hal-01404383/

7. Il s'agit de l'intervention de Adrien Torres Astaburuaga et de Nicolas Tixier, intitulée «Mémoire naturelle, statigraphie urbaine, réactivation par l'usage - Geddes in India, in Valencia ». Consultable sur : https://hal.archives-ouvertes.fr/hal-01404378/

8. Il s'agit de l'intervention de Olfa Meziou intitulée «Les insulations de Sloterdijk - Quelles portées ambiantales et urbaines?». Consultable sur: https://hal.archives-ouvertes.fr/ hal-01414210/

9. Il s'agit de Cristiane Rose Duarte et Ethel Pinheiro, dont la communication s'intitule « Spatial Empathy and urban experience: a case study in a public space from Rio de Janeiro ». Consultable sur : https://hal.archives-ouvertes.fr/hal-01414016/

10. Le congrès propose six sessions en parallèle. Celle dont je parle ici s'intitule « Expériences et expérimentations des ambiances ", elle constitue la session la plus importante en nombre de communications.

11. Il s'agit de «The polysensory dynamics of ambiance - Example of a Japanese garden (Murinan, Kyoto)» de Catherine Szanto. Consultable sur: https://hal.archives-ouvertes.fr/ hal-01414165/

12. Quoique mon parcours, depuis ma formation, sur le terrain des enveloppes ait eu pour effet de me déformater sacrément. 


\section{AUTEUR}

\section{CLOTILDE FÉLIX-FROMENTIN}

Clotilde Felix-Fromentin est ingénieur en chimie-biologie-santé, architecte d'intérieur-designer, et docteur en arts et esthétique. Elle est actuellement chercheur au LaCTH de l'École nationale supérieure d'architecture et de paysage de Lille, et enseignante. L'atelier Ubiloci qu'elle a fondé en 2003 a travaillé une dizaine d'années dans la scénographie scientifique et culturelle, puis l'hôtellerie et l'habitation, avant de muter en « laboratoire de confection d'architecture » au service d'une recherche exploratoire et théorique interrogeant, via la notion d'enveloppe, la poétique de l'habiter (cf. http://www.ubiloci.fr).

clotilde.felixfromentin@gmail.com 